\title{
The role of CD154-CD40 versus CD28-B7 costimulatory pathways in regulating allogeneic Th1 and Th2 responses in vivo
}

\author{
Koji Kishimoto, ${ }^{1}$ Victor M. Dong, ${ }^{1}$ Shohreh Issazadeh, ${ }^{2}$ Eugenia V. Fedoseyeva, ${ }^{3}$ \\ Ana Maria Waaga, ${ }^{1}$ Akira Yamada, ${ }^{1,4}$ Masayuki Sho, ${ }^{1}$ Gilles Benichou, ${ }^{3}$ \\ Hugh Auchincloss, Jr., ${ }^{1,4}$ Michael J. Grusby, ${ }^{5}$ Samia J. Khoury, ${ }^{2}$ and Mohamed H. Sayegh ${ }^{1,6}$
}

${ }^{1}$ Laboratory of Immunogenetics and Transplantation, Brigham and Women's Hospital,

${ }^{2}$ Center for Neurologic Diseases, Brigham and Women's Hospital,

${ }^{3}$ Cellular and Molecular Immunology Laboratory, Schepens Eye Research Institute, Brigham and Women's Hospital, and

${ }^{4}$ Transplantation Unit, Massachusetts General Hospital; Brigham and Women’s Hospital,

Harvard Medical School, Boston, Massachusetts, USA

${ }^{5}$ Department of Immunology and Infectious Disease, Harvard School of Public Health, Boston, Massachusetts, USA

${ }^{6}$ Division of Nephrology, Children's Hospital, Harvard Medical School, Boston, Massachusetts, USA

Address correspondence to: Mohamed H. Sayegh, Laboratory of Immunogenetics and Transplantation, Brigham and Women's Hospital, Harvard Medical School, 75 Francis Street, Boston, Massachusetts 02115, USA. Phone: (617) 732-5259; Fax: (617) 732-5254; E-mail: msayegh@rics.bwh.harvard.edu.

Received for publication February 10, 2000, and accepted in revised form May 31, 2000.

We used signal transducer and activator of transcription 4 (STAT4) and STAT6 gene knockout (-/-) mice as recipients of fully mismatched cardiac allografts to study the role of T-cell costimulatory pathways in regulating allogeneic T-helper 1 (Th1) versus Th2 responses in vivo. STAT4-/- mice have impaired Th1 responses, whereas $S T A T 6^{-/-}$mice do not generate normal Th2 responses. Cardiac allografts from C57BL/6 mice were transplanted into normal wild-type (WT), STAT4-/, and STAT6 ${ }^{-/}$ $\mathrm{BALB} / \mathrm{c}$ recipients. STAT4 $4^{-/}$and $S T A T 6^{-/-}$mice rejected their grafts with the same tempo as untreated WT recipients. CD28-B7 blockade by a single injection of CTLA4Ig induced long-term engraftment and donor-specific tolerance in all three groups of recipients. CD154 blockade by a single injection of MR1 was effective in prolonging allograft survival and inducing tolerance in $S T A T 4^{-/}$mice but was only marginally effective in $S T A T 6^{-/-}$recipients and WT controls. In addition, a similar protocol of MR1 was ineffective in prolonging graft survival in $\mathrm{CD} 28^{-/-} \mathrm{BALB} / \mathrm{c}$ recipients, suggesting that the lack of efficacy seen in WT and STAT6 $6^{-/-}$mice is not due to the presence of a functional CD28-B7 pathway. Furthermore, there was a similar differential effect of CD28-B7 versus CD154-CD40 blockade in inhibiting immune responses in animals immunized with ovalbumin and complete Freund's adjuvant. These novel data indicate that Th1 and Th 2 cells are differentially regulated by CD28-B7 versus CD154-CD40 costimulation pathways in vivo and may have potential implications for the development of therapeutic strategies such as T-cell costimulatory blockade in humans.

J. Clin. Invest. 106:63-72 (2000).

\section{Introduction}

CD4 $4^{+} \mathrm{T}$-helper (Th) cells may be divided into at least two functionally distinct subsets $(1,2)$. Th1 cells are important in cell-mediated immunity, whereas Th2 cells are regulators of the humoral immune response and suppress Th1 cell function (2). In transplantation models, it is traditionally thought that Th1 cells cause acute rejection and Th2 cells induce and maintain tolerance. This paradigm is supported by reports demonstrating Th2 cytokine expression in grafts of tolerant animals (3-7) and Th1 cytokine reversal of the induction of tolerance $(8,9)$. However, there are recent data that challenge this paradigm. For example, rejection occurs in Th1 cytokine-knockout animals $(10,11)$, and tolerance can be induced in Th2 cytokine knockouts $(12,13)$, although this is not a universal observation (14).
It has been suggested that there are several factors that determine the fate of a naive $\mathrm{Th}$ cell in response to antigenic challenge $(15,16)$. These include the strength of the T-cell receptor (TCR) signal or antigen density, the cytokine milieu, and specific costimulatory pathways. T cells require two distinct signals for full activation (17). The first signal is provided by the engagement of the TCR with the major histocompatibility complex (MHC) plus peptide complex on antigen-presenting cells (APCs), and the second "costimulatory" signal is provided by engagement of one or more T-cell surface receptors with their ligands on APCs (15-18). Among the multiple costimulatory pathways identified, increasing evidence suggests that interactions of T-cell surface receptors CD28 and CD154, with their respective ligands B7-1/B7-2 and CD40, on APCs are critical for the T-cell responses to 
alloantigens $(17,19)$. At present, the role of CD28-B7 versus CD154-CD40 in regulating Th1 versus Th2 allogeneic responses in vivo is unknown.

Members of the signal transducer and activator of transcription (STAT) gene family are critical for the differentiation of Th-cell subsets. It has been demonstrated that STAT4 knockout (-/-) mice have impaired generation of Th1 cells, whereas STAT6 ${ }^{-/}$mice do not generate normal Th2 responses $(20,21)$. Therefore, these gene-knockout mice may be used to study the role of Th1 and Th2 responses in rejection and tolerance induction. Using STAT4 ${ }^{-/}$and STAT6 ${ }^{-1-}$ mice as recipients of vascularized, fully mismatched cardiac allografts, we studied the role of T-cell costimulatory pathway blockade with cytotoxic T-lymphocyte antigen 4 (CTLA4Ig; to block CD28-B7) or anti-CD154 mAb (MR1; to block CD154-CD40) in preventing rejection and inducing tolerance in a predominant Th 1 versus Th2 environment. Our data indicate that even in the absence of normal Th1 or Th2 responses, rejection proceeds with the same tempo and that alloreactive Th1 and Th2 cells are differentially regulated by CD28-B7 versus CD154-CD40 costimulatory pathways in vivo.

\section{Methods}

Mice. C57BL/6 $\left(\mathrm{H}-2^{\mathrm{b}}\right)$ and $\mathrm{C} 3 \mathrm{H} / \mathrm{He}\left(\mathrm{H}-2^{\mathrm{k}}\right)$ mice aged 6-8 weeks were purchased from the Jackson Laboratory (Bar Harbor, Maine, USA) and BALB/c $\left(\mathrm{H}-2^{\mathrm{d}}\right)$ mice aged 6-8 weeks were purchased from Taconic Farms (Germantown, New York, USA). CD28-/ $\left(\mathrm{H}-2^{\mathrm{d}}\right)$ mice were purchased from the Jackson Laboratory and bred in our animal facility. Mice homozygous for a targeted disruption of STAT4 gene (STAT4-/- mice) and STAT6 gene (STAT6 ${ }^{-1-}$ mice) have been described previously $(20,21)$. Briefly, IL-12-induced increases in the production of IFN- $\gamma$ cellular proliferation and natural killer (NK) cell cytotoxicity are abrogated in lymphocytes from STAT4-deficient mice. These lymphocytes also demonstrate a propensity toward the development of Th2 cells. On the other hand, IL-4-induced increases in the cell-surface expression of both major histocompatibility complex (MHC) class II antigens and IL4 receptor are completely abrogated, and lymphocytes from STAT6-deficient animals fail to differentiate into Th2 cells in response to either IL- 4 or IL-13.

Fusion proteins and $m A b$ s. MR1 was purchased from Bioexpress Cell Culture Services (West Lebanon, New Hampshire, USA). Murine CTLA4Ig was a generous gift from Bristol Myers Squibb (Robert Peach, Princeton, New Jersey, USA).

Transplantation. BALB/c mice (WT, STAT4-/-, STAT6-/, and $\left.\mathrm{CD} 28^{--}\right)$were used as recipients and $\mathrm{C} 57 \mathrm{BL} / 6$ mice as donors. The first cardiac allografts were placed in an intra-abdominal location (22), and the second heart grafts from $\mathrm{C} 57 \mathrm{BL} / 6$ or $\mathrm{C} 3 \mathrm{H} / \mathrm{He}$ mice were placed in the neck using a modification of Chen's method (23). Graft function was assessed daily by palpation. Animals received a single dose of $\mathrm{mAb}$ or fusion protein at a dose of $250 \mu \mathrm{g}$ by intraperitoneal injection either on the day of transplantation (for MR1) or 2 days after engraftment (for CTLA4Ig). These administration schedules were based on our previous observations that a single injection of CTLA4Ig is most effective when administered on post-transplant day 2 , whereas MR1 is best given on the day of transplant $(4,6,24)$. In some instances, animals also received an intravenous injection of $5 \times 10^{6}$ donor splenocytes at the time of transplantation, as described previously (6). The day of rejection was defined as the day of cessation of palpable heartbeat and was verified by autopsy and selective pathological examination. Loss of graft function within 48 hours of transplant was considered a technical failure ( $<10 \%$ on average), and these animals were excluded from further analysis.

Statistics. For the graft survival, Kaplan-Meier survival graphs were constructed and log-rank comparison of the groups was used to calculate $P$ values. For the ELISPOT results, $P$ values were calculated using the paired $t$ test.

Morphology. Cardiac grafts from both the isografts and each untreated recipient group $(n=3-4)$ were fixed in $10 \%$ buffered formalin, embedded in paraffin, coronally sectioned, and stained with hematoxylin and eosin (H\&E) for evaluation of cellular infiltrates using light microscopy. Cardiac grafts from long-term survivors (>100 days) treated with either MR1 or CTLA4Ig were similarly prepared and stained with H\&E, Verhoeff's elastin (for vessel arteriosclerosis scoring), or Masson's trichrome (for evaluation of fibrosis).

Arteriosclerosis was assessed using light microscopy and percentage luminal occlusion by intimal thickening determined using the scoring system as described previously (25-27). In brief, a vessel score of 0 indicated less than $10 \%$ luminal occlusion, 1 indicated less than 20\%, 2 indicated 20-40\%, 3 indicated 40-60\%, 4 indicated $60-80 \%$, and 5 indicated $100 \%$ occlusion. Only vessels that were cut orthogonally and that displayed a clear internal elastic lamina were scored. All arteries were scored by two blinded examiners.

Matched trichrome- and H\&E-stained sections were also examined for presence for fibrosis and interstitial cellular infiltration. Each of these categories was graded and the average grade calculated for each group. Fibrosis was quantified by collagen deposition highlighted by trichrome stain and scored on a $0-3$ scale, where 0 indicated no fibrosis and 1, 2, and 3 indicated mild, moderate, and severe fibrosis, respectively. A 0-3 scale was also used for scoring the degree of interstitial mononuclear cell infiltration, with $0,1,2$, and 3 indicating normal, minimal, moderate, and severe cellular infiltration, respectively.

ELISPOT cytokine measurement. ELISPOT plates (Polyfiltronics Inc., Rockland, Massachusetts, USA) were coated with either $4 \mu \mathrm{g} / \mathrm{mL}$ of rat anti-mouse IFN- $\gamma$ (R4-6A2) or with $5 \mu \mathrm{g} / \mathrm{mL}$ of rat anti-mouse IL-5 (TFRK-4) capturing mAbs in sterile PBS overnight. The plates were then blocked for 1.5 hours with sterile PBS containing $1 \%$ BSA and washed with sterile PBS. Splenocytes $\left(1.2 \times 10^{6}\right.$ in 0.2 $\mathrm{mL}$ AIM-V medium) were then placed in each well in the 


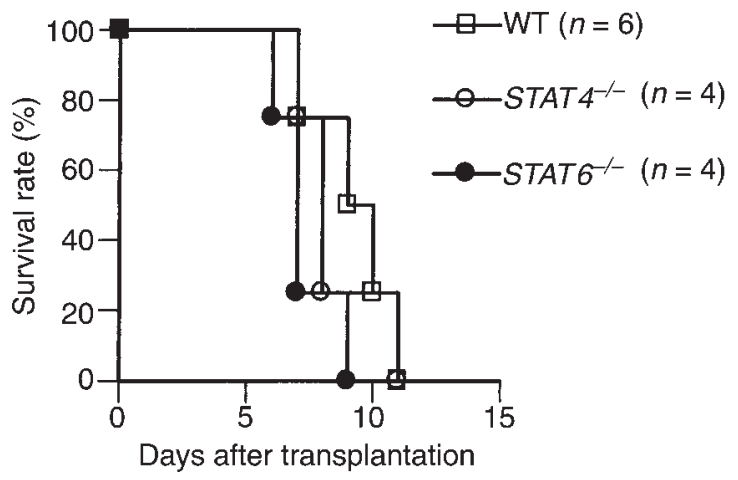

Figure 1

Allograft survival in untreated recipients. C57BL/ 6 hearts were transplanted into wild-type, STAT4-/-, or STAT6 ${ }^{-/-}$BALB/c recipients.

presence of $1.2 \times 10^{6}$ irradiated ( $20 \mathrm{~Gy}$ ) syngeneic or allogeneic splenocytes (as APCs) and cultured for 48 hours at $37^{\circ} \mathrm{C}$ in $5 \% \mathrm{CO}_{2}$. For detection of spots, $5 \mu \mathrm{g} / \mathrm{mL}$ of biotinylated rat anti-mouse IFN- $\gamma$ mAb (XMG 1.2) or rat anti-mouse IL- $5 \mathrm{mAb}$ (TFRK-5) were used, followed by 2 hours of incubation with streptavidin $\mathrm{D}$ horseradish peroxidase (Vector Laboratories, Burlingame, California, USA) diluted at 1:2000 in PBS 0.025\% Tween. All mAbs were purchased from PharMingen (San Diego, California, USA). After washing, the plates were developed using 0.8 $\mathrm{mL}$ of 3-amino-9-ethylcarbazole (AEC) (Pierce Chemical Co., Rockford, Illinois, USA; $10 \mathrm{mg}$ dissolved in $1 \mathrm{~mL}$ dimethyl formamide) mixed with $24 \mathrm{~mL}$ of $0.1-\mathrm{M}$ sodium acetate, $\mathrm{pH}$ 5.0, containing $12 \mu \mathrm{L} \mathrm{H}_{2} \mathrm{O}_{2}$. The resulting spots were counted on a computer-assisted enzyme-linked immunospot image analyzer (T Spot Image Analyzer; Cellular Technology Ltd., Cleveland, Ohio, USA) (28).

Immunization with ovalbumin and cell preparation for in vitro assay. The animals were immunized with $100 \mu \mathrm{L}$ of ovalbumin (OVA)/CFA subcutaneously, such that each mouse received a total of $100 \mu \mathrm{g}$ of OVA, as described previously (29). Treatment was started with CTLA4Ig (100 $\mu \mathrm{g}$ per dose) or MR1 (100 $\mu$ g per dose) on the day of immunization and given every other day for five doses. Control mice received control Ig. Lymph nodes were collected on day 10 after immunization. A single-cell suspension was prepared from draining popliteal lymph nodes for measurement of proliferation and cytokine production (29).

Proliferation and cytokine-production assays. Proliferation and cytokine production were measured after stimulation with OVA in vitro at three doses $(25,50$, and $100 \mu \mathrm{g} / \mathrm{mL}$ ), as described previously (29). For proliferation and cytokine measurement, the cells were cultured in 96-well plates (Corning-Costar, Cambridge, Massachusetts, USA) with serum-free Ex Vivo 20 medium (BioWhittaker, Walkersville, Maryland, USA) containing $75 \mathrm{mM} / \mathrm{mL}$ L-glutamine, $100 \mathrm{U} / \mathrm{mL}$ penicillin and streptomycin, $1 \mathrm{~mL} / 100 \mathrm{~mL}$ media of a $100 \times$ concentrated, nonessential amino acid solution, $0.1 \mathrm{mM}$ HEPES $/ \mathrm{mL}, 1 \mathrm{mM} / \mathrm{mL}$ sodium pyruvate (all from BioWhittaker), and $0.05 \mathrm{mM} / \mathrm{mL} 2$-mercaptoethanol (Sigma Chemical Co., St. Louis, Missouri, USA). The cells were incubated at $37^{\circ} \mathrm{C}$ in humidified air containing $7 \% \mathrm{CO}_{2}$.

For proliferation, lymph node cells were cultured at 2 $\times 10^{6}$ cells $/ \mathrm{mL}$ and $200 \mu \mathrm{L} /$ well with various antigen concentrations. After 96 hours of culture $1 \mu \mathrm{Ci}{ }^{3} \mathrm{H}$ thymidine (NEN Life Sciences Products, Boston, Massachusetts, USA) was added in $10 \mu \mathrm{L}$ of media to each well for another 16 hours. Cells were harvested on filter mats using a TOMTEC harvester and incorporation of thymidine was analyzed with a Betaplate counter (PerkinElmer Wallac Inc., Gaithersburg, Maryland, USA).

For cytokine assays, the cells were cultured at $2 \times 10^{6}$ cells $/ \mathrm{mL}$ in $200 \mu \mathrm{L}$ media at various antigen concentrations. Supernatants for IL- 4 and IFN- $\gamma$ ELISA were collected after 48 hours of culture. Quantitative ELISAs for IL-4 and IFN- $\gamma$ were performed using paired anti-
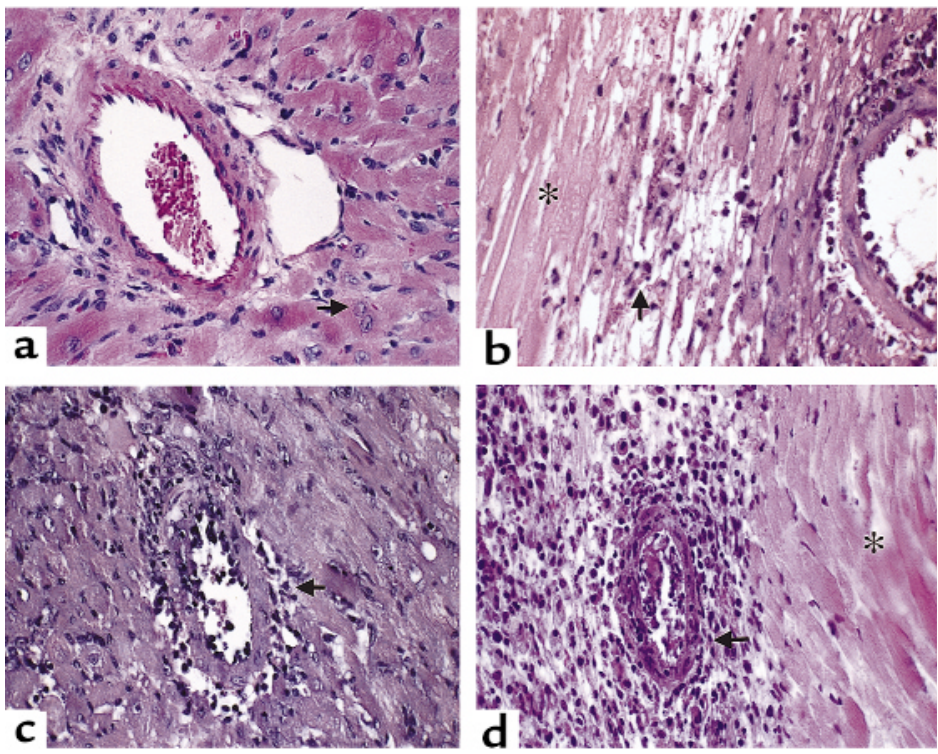

\section{Figure 2}

Pathology of murine cardiac allograft rejection: syngeneic graft at day 8 after transplantation and allografts of WT, STAT4 $4^{-/}$, and STAT6 ${ }^{-/-}$recipients. (a) Syngeneic graft: the artery shows no signs of inflammation and the cardiac muscle cells are intact, with viable nuclei (arrow). (b) Wild-type recipient graft: the small artery reveals inflammatory cells adherent to the endothelium. There is extensive infarction (coagulative or ischemic necrosis) of cardiac muscle cells (asterisk) and mononuclear inflammatory cell infiltration of the viable myocardium (arrow). (c) STAT4 ${ }^{-1-}$ recipient graft: there is active inflammation or endothelialitis of the inner layer of the small artery (arrow). The myocardium shows scattered lymphocytes. (d) STAT6 ${ }^{-1-}$ recipient graft: the active transmural inflammation (arrow) has resulted in extensive infarction of the myocardium (asterisk). There is also prominent inflammation of the parenchyma. Original magnification $\times 400(\mathrm{H} \& \mathrm{E})$. 


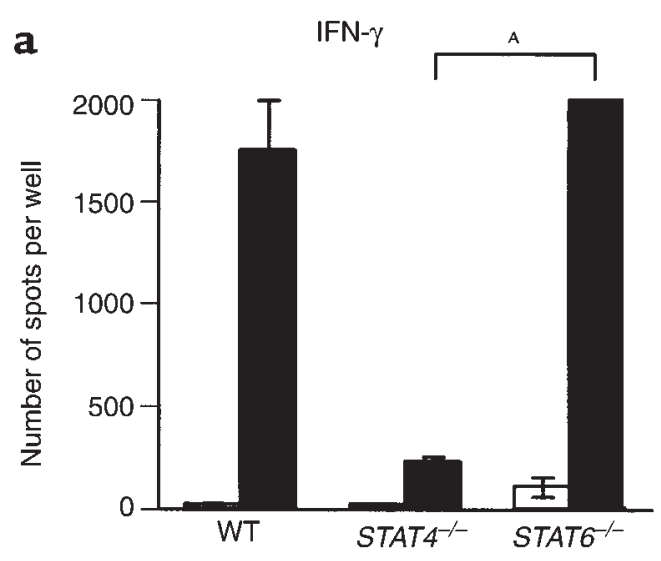

b

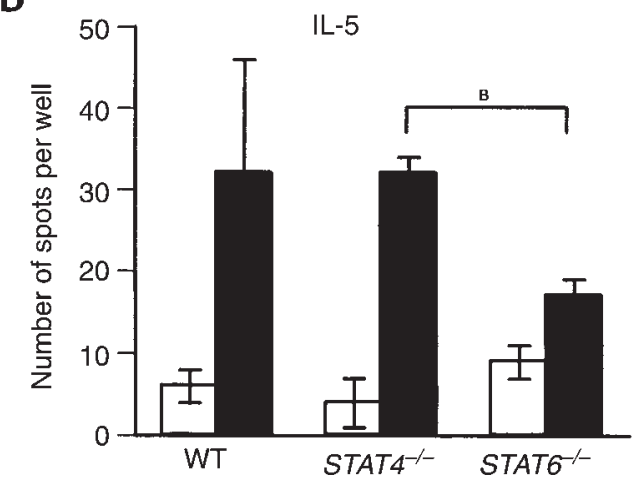

Figure 3

Frequency of cytokine-producing donor-specific $T$ cells in recipient WT, STAT4-/-, and STAT6 ${ }^{--}$BALB/c mice following allogeneic heart transplantation. Mice were transplanted with $\mathrm{C} 57 \mathrm{BL} / 6$ allogeneic hearts. Recipient spleens were removed at the time of rejection (9-10 days after transplant). Splenocytes $\left(1.2 \times 10^{6}\right.$ cells per well) were incubated with either recipient (open bars) or donor (filled bars) irradiated splenocytes. The frequencies of IFN- $\gamma$-producing $T$ cells $(\mathbf{a})$ and IL-5-producing T cells (b) were then determined using ELISPOT assay as described in Methods. The results are expressed as the mean number of spots per well \pm SE obtained from three to four mice tested individually in each group. $P$ values of syngeneic versus alloreactive responder frequencies in each animal group are as follows. IFN- $\gamma: P=$ $0.0196,0.0132$, and 0.006 in WT, STAT4 ${ }^{-{ }^{--}}$, and STAT6 ${ }^{-/-}$recipients, respectively. IL-5: $P=0.2327,0.0092$, and 0.0942 in WT, STAT4 ${ }^{-1-}$, and $S T A T 6^{-/-}$recipients, respectively. ${ }^{A} P=0.002 ;{ }^{B} P=0.0493$.

bodies and recombinant cytokines from PharMingen according to the manufacturer's recommendations.

\section{Results}

Effect of cytokine milieu on acute cardiac allograft rejection. First, we investigated the effect of the absence of either the STAT4 or STAT6 signal transduction pathway on rejection of fully mismatched cardiac allografts. As shown in Figure 1, both STAT4- and STAT6- recipients were able to acutely reject their vascularized cardiac allografts; there was no significant difference in graft survival times between WT versus either STAT4-or $S T A T 6^{-/}$recipients. We then examined the graft morphology of the rejecting recipients. Sections of cardiac allografts were stained with $\mathrm{H} \& \mathrm{E}$ and were examined under light microscopy. Four groups of animals were studied: $\mathrm{BALB} / \mathrm{c}$ into $\mathrm{BALB} / \mathrm{c}, \mathrm{C} 57 \mathrm{BL} / 6$ into WT $\mathrm{BALB} / \mathrm{c}, \mathrm{C} 57 \mathrm{BL} / 6$ into STAT4- $\mathrm{BALB} / \mathrm{c}$, and $\mathrm{C} 57 \mathrm{BL} / 6$ into $S T A T 6^{--} \mathrm{BALB} / \mathrm{c}$. Syngeneic functioning control grafts demonstrated preservation of myocytes with intact nuclei and absence of interstitial cellular infiltrates or vascular inflammation of cardiac vessels (Figure 2a). Rejected grafts from all three experimental recipient groups (WT, STAT4--, and STAT6--) demonstrated variable degrees of diffuse mononuclear cellular infiltration, endothelialitis, and areas of myocyte damage and interstitial infarction (Figure 2, b-d). These pathologic observations confirm the in vivo graft-survival data and clearly demonstrate that vascularized rejection occurs in the absence of normal Th1 or Th2 allogeneic responses.

Cytokine profiles of untreated WT, STAT4-, and STAT6-recipients in response to donor alloantigen. We then investigated profiles of cytokines produced by untreated recipients in response to donor alloantigen. ELISPOT assay was used to compare cytokine production (IFN- $\gamma$ as a prototype Th1 cytokine and IL-5 as a prototype Th2 cytokine) by splenocytes from untreated WT, STAT4-\%, or $S T A T 6^{-/}$recipients that had acutely rejected heart allografts in response to donor or syngeneic (negative control) stimulator cells in vitro. As shown in Figure 3a, the frequencies of alloreactive IFN- $\gamma$-producing recipient cells were significantly higher than autoreactive frequencies in all groups, although the alloreactive frequency was significantly lower $(P=0.002$; difference between the means was 1769.7) for STAT4- as compared with $S T A T 6^{--}$mice. In comparison, the alloreactive frequency of IL-5-producing cells was only significantly higher than the autoreactive frequency in STAT4- recipients (Figure $3 \mathrm{~b}$ ), and that was significantly higher $(P=0.0493$; difference between the means was 17) than the alloreactive frequency of STAT6- mice. WT recipients demonstrated some variability of IL-5 production in response to allogeneic cells (Figure 3b).

Effect of T-cell costimulatory signal blockade on cardiac allograft rejection in WT recipients. BALB/c mice were transplanted with $\mathrm{C} 57 \mathrm{BL} / 6$ cardiac allografts and treated with CTLA4Ig or MR1. Whereas a single injection of CTLA4Ig on post-transplant day 2 markedly prolonged graft survival, a single injection of MR1 alone administered on day 0 was significantly less effective (Figure 4a). This observation with MR1 was not strain specific since a single injection of MR1 had a comparable effect in C57BL/ 6 recipients of $B A L B / c$ hearts (survival times: $8,41,41,52$, $>100 \times 2 ; P=\mathrm{NS}$ ) as compared with $\mathrm{BALB} / \mathrm{c}$ recipients of C57BL/ 6 hearts. Donor-specific tolerance induction by CTLA4Ig treatment was confirmed by secondary heartgraft challenge of animals with long-term surviving grafts (>100 days). Whereas third-party $(\mathrm{C} 3 \mathrm{H} / \mathrm{He})$ second grafts were rejected, grafts from the original donor strain were permanently accepted (Table 1).

The observation that MR1 treatment was less effective than CTLA4Ig could be attributed to the continued presence of costimulation through the CD28-B7 path- 
a

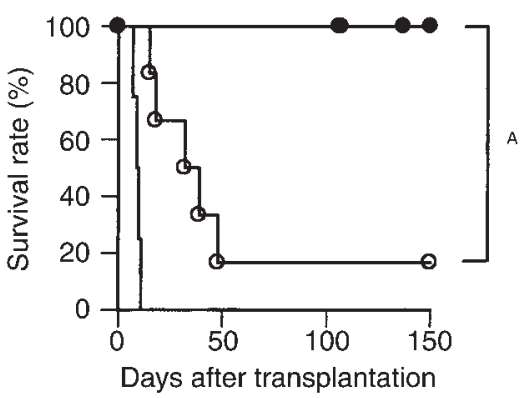

No treatment $(n=7) \multimap$ MR1 $(n=6) \longrightarrow$ CTLA4lg $(n=10)$

b

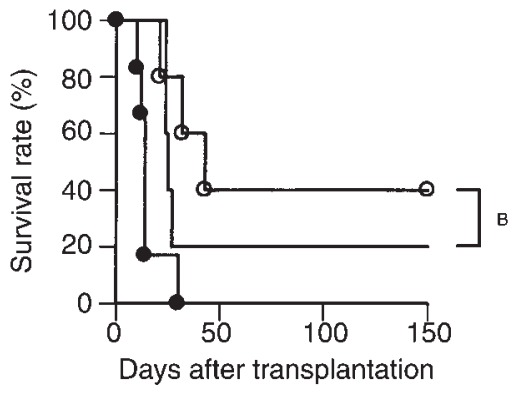

No treatment $(n=6) \multimap$ MR1 $(n=6) \longrightarrow$ CTLA4lg $(n=6)$

\section{Figure 4}

Effect of MR1 or CTLA4lg treatment on cardiac allograft survival in wild-type (a) or $\mathrm{CD}_{28^{-/-}}$mice (b). Recipients were treated with MR1 (250 $\mu \mathrm{g}$ intraperitoneally) on the day of transplantation or with CTLA4Ig ( $250 \mu \mathrm{g}$ intraperitoneally) on day 2 . Untreated controls are shown by solid line. ${ }^{A} P<0.01 ;{ }^{B} P=0.33$.

way. To investigate this possibility, we administered a single injection of $250 \mu \mathrm{g} \mathrm{MR} 1$ to $\mathrm{CD} 28^{-/}$allograft recipients. As shown in Figure $4 \mathrm{~b}$, untreated $C D 28^{-}$ recipients had some prolongation of graft survival as reported previously (30), but MR1 treatment was ineffective in promoting long-term graft acceptance, suggesting that even in the absence of a functional CD28B7 signal, CD154 blockade did not induce tolerance. Effect of T-cell costimulatory signal blockade on cardiac allograft rejection in STAT4-> recipients. Similar to our findings in WT recipients, CTLA4Ig treatment prolonged survival and induced donor-specific tolerance to cardiac allografts in STAT4- recipients (Figure 5 and Table 1). Interestingly, unlike the case of WT recipients, MR1 promoted long-term graft survival and induced donor-specific tolerance (acceptance of second-donor strain grafts and rejection of third-party grafts; Table 1) in STAT4-- recipients similar to CTLA4Ig therapy. These data suggest that CD154 blockade is more effective in suppressing alloimmune responses in the absence of a normal Th1 cytokine milieu.

Effect of T-cell costimulatory signal blockade on cardiac allograft rejection in STAT6--recipients. We then investigated the effect of CD28-B7 versus CD154-CD40 blockade on fully mismatched cardiac allograft rejection using STAT6 ${ }^{-/}$recipients. Treatment with CTLA4Ig prolonged graft survival and induced donor-specific toler- ance as seen in WT and STAT4-- recipients, whereas therapy with MR1 was significantly less effective in STAT6 $6^{-}$recipients, similar to our observations in WT mice (Figure 6 and Table 1). Overall, CD154 blockade was significantly more effective in promoting longterm graft survival in STAT4- recipients when compared with either WT $(P=0.0438)$ or STAT6 $\sigma^{-}$recipients $(P=0.0461)$. These data indicate that CD154 blockade is only marginally effective in suppressing alloimmune responses in a predominantly Th1 environment.

Effect of CD28-B7 versus CD154-CD40 blockade on development of chronic rejection. Grafts of WT, STAT4--, or $S T A T 6^{--}$recipients treated with a single injection of MR1 or CTLA4Ig surviving more than 100 days were evaluated histopathologically for evidence of chronic rejection as manifested by graft arteriosclerosis, fibrosis, and interstitial inflammation. Grafts of animals treated with CTLA4Ig had minimal signs of arteriosclerosis (vessel scores were less than 0.5 in all groups), interstitial inflammation, or fibrosis. Grafts of animals treated with MR1, on the other hand, developed moderately severe arteriosclerosis (mean vessel scores of WT, STAT4-/, and STAT6-- recipients treated with MR1 were 3, $3.10 \pm 1.07$, and $3.25 \pm 1.75$, respectively), fibrosis, and interstitial inflammation. Although, as indicated above, the number of longterm survivors were small in the WT and STAT6-recipients treated with MR1 (1-2/group), there did not appear to be any difference in the pathology of chronic rejection between the different recipient groups treated with MR1.

Differential effects of CD28-B7 and CD154-CD40 blockade in priming of STAT4-- and STAT6-- mice to nominal antigen. To determine if the differential effects of CD28-B7 versus CD154-CD40 blockade in regulating Th1 and Th2 responses are applicable to a nominal antigen system, we studied the immune response of WT, STAT4--, and $S T A T 6^{-}-\mathrm{BALB} / \mathrm{c}$ mice immunized with OVA/CFA. We treated WT, STAT4- , and STAT6-- BALB/c mice with CTLA4Ig or MR1 in vivo. In vitro stimulation of primed lymph node cells with OVA showed similar proliferative responses in control Ig-treated WT, STAT4--, and STAT6 $6^{->}$mice. Whereas CTLA4Ig treatment suppressed the proliferative response in all treated mice, MR1 treatment was only effective in suppressing proliferation in STAT4-- mice (Figure 7a). Cytokine pro-

\section{Table 1}

Second heart graft survival (days) in animals bearing original heart graft for more than 85 days

\begin{tabular}{lccc}
\hline \multirow{2}{*}{ Recipient } & Original treatment & \multicolumn{2}{c}{ Second graft donor } \\
& & C57BL/6 & $\mathrm{C} 3 \mathrm{H} / \mathrm{He}$ \\
WT & CTLA4Ig & $>100,>100,>100$ & $9,13,35$ \\
STAT4 ${ }^{-1}$ & CTLA4Ig & $>61,>89,>100$ & 8,8 \\
STAT4 $^{-1}$ & MR1 & $>100,>100$ & 13 \\
STAT6- $^{--}$ & CTLA4Ig & $>55,>100,>100$ & $9,36,36$ \\
\hline
\end{tabular}




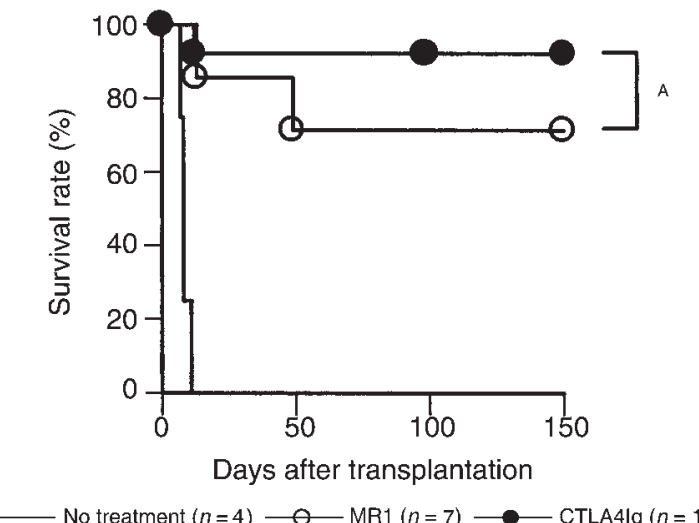

Figure 5

Effect of MR1 or CTLA4Ig treatment on cardiac allograft survival in STAT4 ${ }^{-/}$mice. Recipients were treated with MR1 $(250 \mu \mathrm{g}$ intraperitoneally) on the day of transplantation or with CTLA4Ig ( $250 \mu \mathrm{g}$ intraperitoneally) on day 2 . Untreated controls are shown by solid line. ${ }^{A} P=0.14$.

duction was also measured, and the results of both IFN- $\gamma$ and IL- 4 paralleled those of the proliferation assay. In STAT4-- mice, a small amount of IFN- $\gamma$ was produced, and treatment with MR1 abrogated it completely (Figure $7 \mathrm{~b}$ ). In addition, IL-4 production in STAT4- mice was suppressed after MR1 treatment as well as after CTLA4Ig treatment. As expected, there was minimal IL-4 detectable in the supernatants of the STAT6-- mice cultures, but similar to IFN- $\gamma$ production, it was unchanged after MR1 treatment (Figure 7c). These findings underscore the fact that the differential effects of CD154-CD40 in STAT4- versus STAT6- mice are not restricted to allogeneic stimulation but also apply to nominal antigen systems in vivo.

Strategies to optimize the effect of CD154 blockade in WT and STAT6- recipients. First, we investigated whether increasing the frequency of administration of MR1 could further improve graft survival in WT and STAT6- recipients. As shown in Figure 8, administration of four doses of MR1 $(250 \mu \mathrm{g}$ on post-transplant day $0,2,4$, and 6) significantly improved graft survival in WT and STAT6- recipients as compared with the single injection protocol. In addition, we $(6,31)$ and others (32) have shown previously that administration of donor cells synergize with CD154 blockade to promote long-term allograft survival. Therefore, we wanted to investigate the effect of combining donor splenocytes plus CD154 blockade in WT, STAT4--, and $S T A T 6^{-/}$recipients of cardiac allografts. All recipients received an intravenous injection of $5 \times 10^{6}$ donor splenocytes plus a single dose of MR1 on the day of transplantation, as above. As seen in Figure 9, coadministration of donor splenocytes plus a single injection of MR1 resulted in long-term graft survival in all WT, STAT4- ${ }^{-}$, and STAT6 $6^{--}$recipients. Therefore, the addition of donor cells rendered CD154 blockade more effective in inhibiting Th1 allogeneic responses in vivo. These data, taken together with the data obtained with the multiple injection protocol, indicate that Th1 cells are not inherently resistant to CD154 blockade.

\section{Discussion}

We used STAT4 - and STAT6- mice to study the role of CD28-B7 and CD154-CD40 costimulatory pathways in regulating Th1 versus Th2 alloimmune responses in vivo. Our data clearly show that clinical and morphological rejection occurs with a normal tempo in recipients lacking normal Th1 (STAT4--) or Th2 responses (STAT6--). To our knowledge, this is the first report describing in vivo studies of knockout animals targeted at the STAT 4 and 6 genes in an organ-transplant model. Our data are consistent with data published previously using single cytokine gene-knockout recipients such as

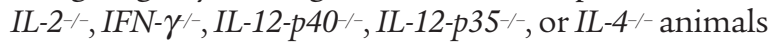
$(10-13,33)$. These data, in concert with our observations, challenge the simplistic traditional Th1/Th2 paradigm in transplantation that Th1 promotes graft rejection and Th2 is permissive for graft acceptance (34-36).

The most important and novel findings of this report are the differential effects of CD28-B7 blockade by CTLA4Ig and CD154-CD40 blockade by MR1 treatment in STAT4-/ versus STAT6- mice. Whereas a single injection of CTLA4Ig on post-transplant day 2 was universally effective in promoting long-term graft survival and inducing donor-specific tolerance in WT, STAT4- , and STAT6 ${ }^{-/}$recipients, a single injection of MR1 was effective primarily in STAT4- mice, but not in WT and $S T A T 6^{--}$mice, in promoting long-term engraftment and tolerance. Our studies with both (a) a multiple-injection protocol of MR1 and (b) a single injection of MR1 with coadministration of donor splenocytes (Figures 8 and 9), clearly indicate that Th1 cells of WT and $S T A T 6^{-/}$recipients are not inherently resistant to CD154 blockade.

Several studies have shown previously that CD28-B7 blockade is effective in blocking Th1 immune responses and in some models may spare Th2 cell function (4,

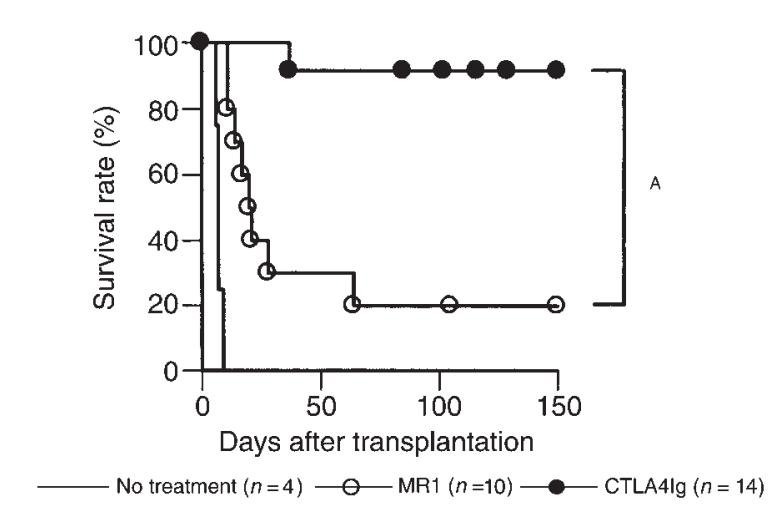

Figure 6

Effect of MR1 or CTLA4Ig treatment on cardiac allograft survival in STAT6 ${ }^{-/}$mice. Recipients were treated with MR1 $(250 \mu \mathrm{g}$ intraperitoneally) at the day of transplantation or with CTLA4Ig ( $250 \mu \mathrm{g}$ intraperitoneally) on day 2 . Untreated controls are shown by solid line. ${ }^{A} P<0.01$. 
a
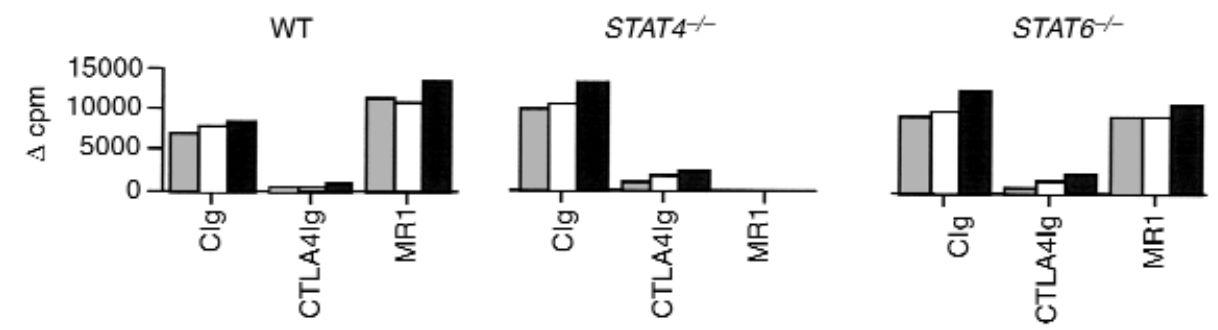

b
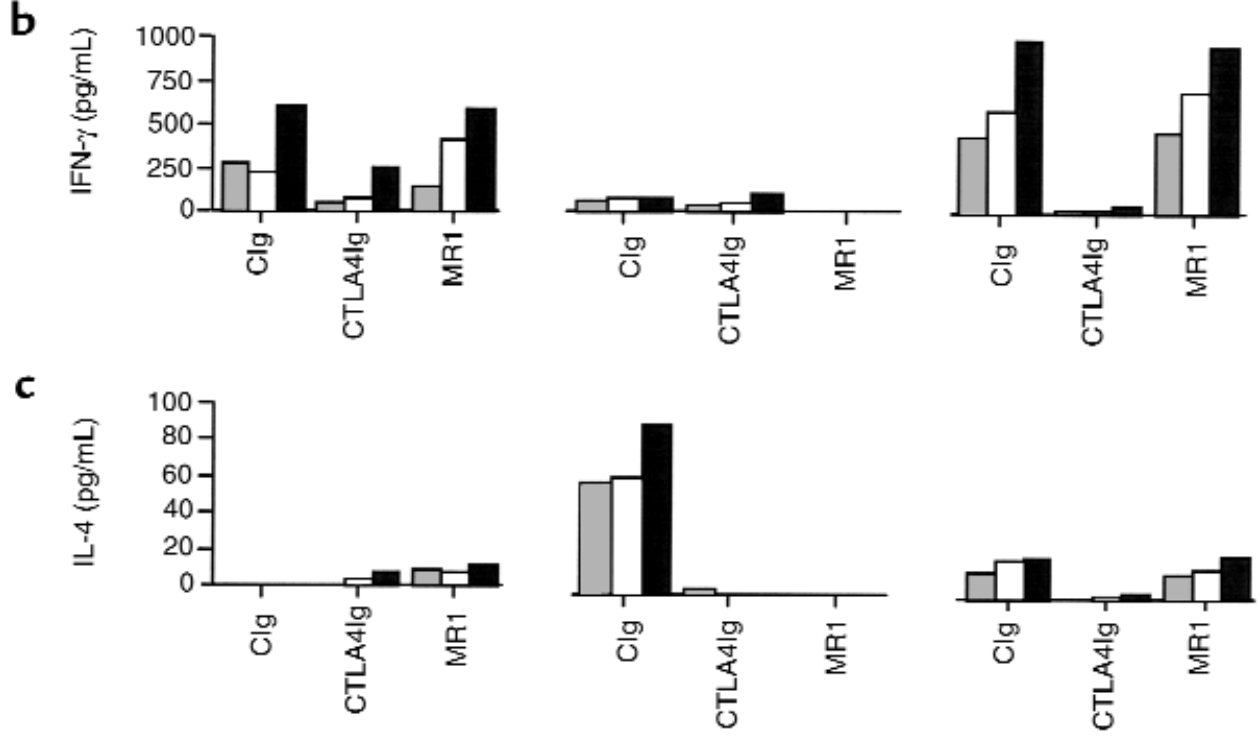

\section{Figure 7}

Effect of MR1 or CTLA4Ig treatment on in vitro lymphocyte proliferation and cytokine production in mice immunized with OVA. The animals were immunized with OVA/CFA, and treatment started with CTLA4Ig or MR1 on the day of immunization and given every other day for five doses. Control mice received control $\lg (\mathrm{Clg})$. Lymph nodes were collected on day 10 after immunization and proliferation (a), and cytokine production (b and $\mathbf{c}$ ) was measured after stimulation with OVA in vitro at three doses: $25 \mu \mathrm{g} / \mathrm{mL}$, shaded bar; $50 \mu \mathrm{g} / \mathrm{mL}$, open bar; and $100 \mu \mathrm{g} / \mathrm{mL}$, filled bar.

$37,38)$. However, there are also data to indicate that CD28 signaling promotes Th2 responses (39) and that CD28-B7 blockade is effective in blocking immune responses in Th2-mediated diseases (40-44). Our data clearly indicate that CTLA4Ig inhibits immune responses in both STAT4- and STAT6/- recipients, suggesting that CD28-B7 blockade is effective in blocking the prevailing immune response irrespective of whether it is predominantly Th1 or Th2. In addition, CTLA4Ig was also effective in preventing development of chronic rejection, confirming our previous studies in rat models of chronic allograft rejection $(26,45,46)$. These data have relevant clinical implications for several immune-mediated human diseases.

Our observations with CTLA4Ig in STAT4- mice are also consistent with data published previously showing that IFN- $\gamma$ is necessary for tolerance induction in a fully allogeneic transplant model (10), since STAT4- - mice produce low levels of IFN- $\gamma$ that is independent of IL-12 signaling $(47,48)$. Our data confirm these observations in an alloantigen as well as a nominal antigen system (see Figure 3a and $7 \mathrm{~b}$ ). These levels may be sufficient for the required putative regulatory functions of IFN- $\gamma(10)$.
The exact mechanisms of the interesting and somewhat surprising data of the differential effect of CD154 blockade with a single injection of MR1 in WT and STAT6- versus STAT4- animals are unknown. Our data with the $C D 28^{-/}$recipients indicate that the marginal efficacy of MR1 in WT and STAT6- recipients is distinctly independent of a functional CD28-B7 pathway. This is also supported by the fact that a similar protocol of MR1 therapy was very effective in STAT4-animals. The fact that CD154 blockade is effective in blocking immune responses in STAT4-- animals is consistent with previous studies establishing the efficacy of such an approach in Th2-mediated diseases like hapten-induced dermatitis (49), Graves' disease (50), and collagen-induced arthritis (51).

The relative resistance of a predominantly Th1 immune response to CD154 blockade in WT and STAT6-- animals have three potential explanations. First, it is possible that CD154-CD40 blockade requires the presence of Th2 cytokines in order to be optimally effective. This is supported by a previous observation from our group that CD154 blockade results in a Th2 switch in a cardiac transplant model $(6,31)$. A second, yet relat- 


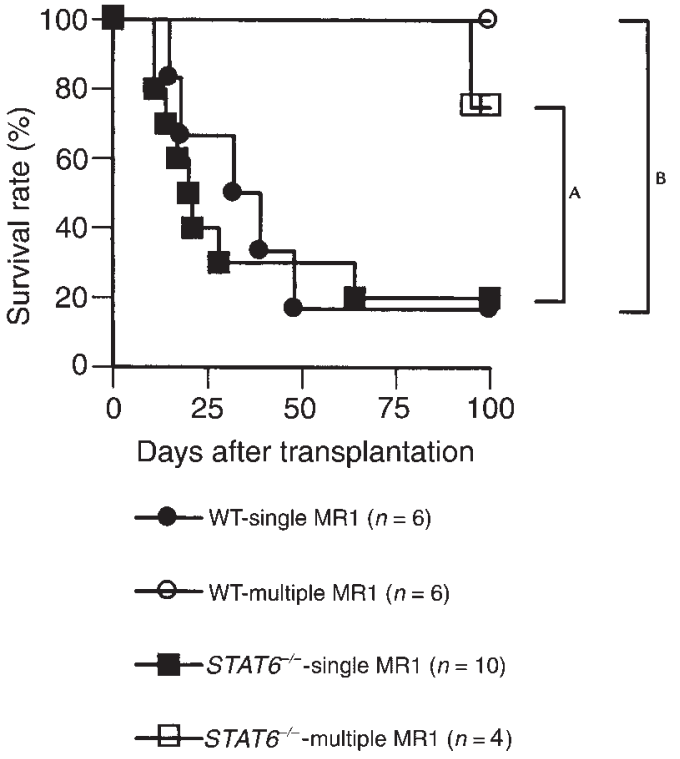

\section{Figure 8}

Effect of administration of multiple injections of MR1 on cardiac allograft survival in WT and STAT6-/- recipients. C57BL/ 6 hearts were transplanted into wild-type or STAT6 ${ }^{-1-}$ BALB/C recipients. Recipients were treated with either a single dose of MR1 alone (250 $\mu \mathrm{g}$ intraperitoneally) on the day of transplantation or four doses of MR1 (250 $\mu \mathrm{g}$ intraperitoneally, four times) on post-transplant day $0,2,4$, and 6 . The multiple-injection protocol significantly increased graft survival in both recipient groups. ${ }^{A} P=0.0456 ;{ }^{B} P=0.0043$.

ed, explanation is the possibility that high precursor frequency of antigen-specific $T$ cells (such as in the transplant setting) or a high antigenic load in the presence of Th1 cytokines is less responsive to CD154 blockade alone. This hypothesis is supported by a recent study by Li et al. (52) that showed Th1 to Th2 immune deviation after anti-IL-12 therapy caused prolonged engraftment in recipients of MHC-matched but minor histocompatibility-antigen mismatched (low alloreactive T-cell mass/low antigen-density system) grafts as compared with recipients of MHC-mismatched (high alloreactive T-cell mass/high antigen-density system) allografts. Third, a predominance of Th1 cytokines may result in priming of effector cells that are resistant to costimulatory blockade. In support of this possibility are the recent findings in some models (53) and in some mousestrain combinations (54), that T-cell costimulatory blockade was not effective in inhibiting rejection driven by $\mathrm{CD}^{+} \mathrm{T}$ cells. Therefore, it is possible that in WT and $S T A T 6^{-/}$recipients a predominance of Th1 cytokines activate $\mathrm{CD} 8^{+} \mathrm{T}$ cells, which may in turn play a critical role in graft rejection and may not be completely blocked by MR1 (see below). Indeed, the ELISPOT data presented in Figure 3 a show a significantly high number of IFN$\gamma$-producing cells in response to alloantigen in vitro in both WT and STAT6-- recipients. Therefore, whereas a single injection of MR1 was insufficient to block this predominant Th1 immune response in vivo, increasing the frequency of administration of MR1 resulted in sig- nificant improvement in graft survival as compared with the single-injection protocol in WT and STAT6/- recipients. Our observations are consistent with data reported by Kirk et al. in primates showing that a short course of anti-CD154 (55) was less effective in promoting longterm rejection-free survival of kidney grafts as compared with chronic anti-CD154 therapy (56). Furthermore, in our model, even in cases where MR1 did result in prolonging graft survival, it was still not as effective as CTLA4Ig in completely inhibiting the alloimmune response to prevent development of chronic rejection. These observations are important for designing clinically relevant strategies based on T-cell costimulatory blockade in organ transplantation.

In several fully allogeneic transplant models, blockade of CD154 alone was ineffective in inducing tolerance; adjunctive therapies with donor antigen in the form of donor cells or bone marrow infusion, or simultaneous blockade of CD28-B7, was necessary to achieve long-term graft acceptance and tolerance $(6,57)$. Our data with donor splenocytes combined with a single injection of MR1 confirm the synergy between administration of donor alloantigens and CD154 blockade in promoting long-term graft survival even in a predominantly Th1 environment that is usually less responsive to CD154 blockade alone (STAT6- recipients). The mechanisms of action of donor alloantigen administration in promoting tolerance remain unknown, although both microchimerism as well as donor-antigen source theories have been proposed $(58,59)$. However, one possibility is that administration of donor splenocytes may act by tolerizing alloreactive $\mathrm{CD}^{+} \mathrm{T}$ cells. Indeed, there are data to indicate that administration of donor alloantigen is effective in tolerizing recipient $2 \mathrm{C}$ transgenic mice (60). These mice have predominantly $\mathrm{CD}^{+} \mathrm{T}$ cells that are reactive to allogeneic class I MHC molecules $\mathrm{L}^{\mathrm{d}}$. Obviously, further studies are required to dissect the exact mechanisms of the

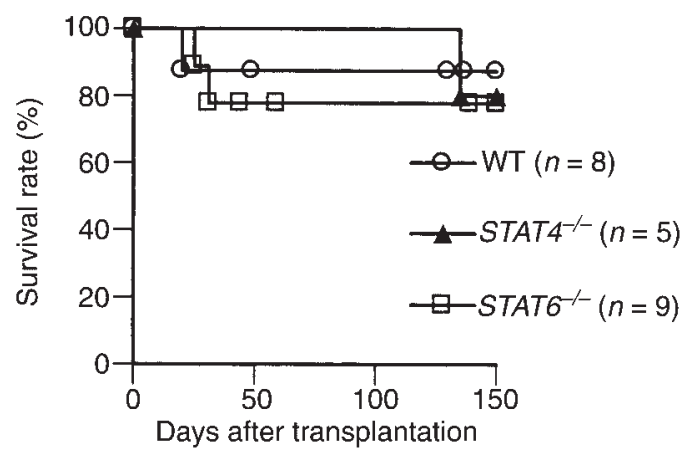

Figure 9

Effect of coadministration of donor splenocytes plus a single injection of MR1 on cardiac allograft survival in WT, STAT4-1-, and STAT6 $^{-/-}$recipients. C57BL/ 6 hearts were transplanted into WT, STAT4 $4^{-/-}$, or STAT6 ${ }^{-1-}$ BALB/c recipients. Recipients were treated with MR1 (250 $\mu$ g intraperitoneally) plus $5 \times 10^{6}$ donor splenocytes intravenously on the day of transplantation. There were no significant differences between the three recipient types. 
observed differential effects of CD154 blockade in a predominantly Th1 versus Th2 environment and the role of donor cells in promoting tolerance.

In conclusion, our data show that whereas CD28-B7 blockade is effective in inhibiting Th1 as well as Th2 alloimmune responses and promoting tolerance in vivo, CD154 is quantitatively less effective in a predominantly Th1 environment. These novel observations have implications for understanding the mechanisms of allograft rejection and tolerance and may impact the development of future immunotherapeutic strategies in transplantation and other immune-mediated diseases.

\section{Acknowledgments}

This work is supported by National Institutes of Health grants RO1 AI-34965 and PO1 AI-41521 (M.H. Sayegh) and AI-43496 (S.J. Khoury). V.M. Dong is a recipient of a National Institutes of Health (National Institute of Diabetes and Digestive and Kidney Diseases) National Research Service Award. Review of photomicrographs by Helmut G. Rennke is greatly appreciated.

1. Mosmann, T.R., Cherwinski, H., Bond, M.W., Giedlin, M.A., and Coffman, R.L. 1986. Two types of murine helper T cell clone. I. Definition according to profiles of lymphokine activities and secreted proteins. $J$. Immunol. 136:2348-2357.

2. Abbas, A.K., Murphy, K.M., and Sher, A. 1996. Functional diversity of helper T lymphocytes. Nature. 383:787-793.

3. Takeuchi, T., Lowry, R.P., and Konieczny, B. 1992. Heart allografts in murine systems. The differential activation of Th2-like effector cells in peripheral tolerance. Transplantation. 53:1281-1294.

4. Sayegh, M.H., et al. 1995. CD28-B7 blockade after alloantigenic challenge in vivo inhibits Th1 cytokines but spares Th2. J. Exp. Med. 181:1869-1874.

5. Binder, J., et al. 1996. The effects of nondepleting CD4 targeted therapy in presensitized rat recipients of cardiac allografts. Transplantation. 61:804-811.

6. Hancock, W.W., et al. 1996. Costimulatory function and expression of CD40 ligand, CD80, and CD86 in vascularized murine cardiac allograft rejection. Proc. Natl. Acad. Sci. USA. 93:13967-13972.

7. Onodera, K., et al. 1997. Type 2 helper T cell-type cytokines and the development of "infectious" tolerance in rat cardiac allograft recipients. J. Immunol. 158:1572-1581.

8. Dallman, M.J., Shiho, O., Page, T.H., Wood, K.J., and Morris, P.J. 1991. Peripheral tolerance to alloantigen results from altered regulation of the interleukin 2 pathway. J. Exp. Med. 173:79-87.

9. Gianello, P.R., et al. 1994. Mechanism of cyclosporin-induced tolerance to primarily vascularized allografts in miniature swine. Effect of administration of exogenous IL-2. J. Immunol. 153:4788-4797.

10. Konieczny, B.T., et al. 1998. IFN-gamma is critical for long-term allograft survival induced by blocking the CD28 and CD40 ligand T cell costimulation pathways. J. Immunol. 160:2059-2064.

11. Dai, Z., Konieczny, B.T., Baddoura, F.K., and Lakkis, F.G. 1998. Impaired alloantigen-mediated $\mathrm{T}$ cell apoptosis and failure to induce long-term allograft survival in IL-2-deficient mice. J. Immunol. 161:1659-1663.

12. Lakkis, F.G., et al. 1997. Blocking the CD28-B7 T cell costimulation pathway induces long term cardiac allograft acceptance in the absence of IL-4. J. Immunol. 158:2443-2448.

13. Mottram, P.L., Raisanen-Sokolowski, A., Glysing-Jensen, T., Stein-Oakley, A.N., and Russell, M.E. 1998. Cardiac allografts from IL-4 knockout recipients: assessment of transplant arteriosclerosis and peripheral tolerance. J. Immunol. 161:602-609.

14. Sirak, J.H., Orosz, C.G., Roopenian, D.C., Wakely, E. and VanBuskirk, A.M. 1998. Cardiac allograft tolerance: failure to develop in interleukin4-deficient mice correlates with unusual allosensitization patterns. Transplantation. 65:1352-1356.

15. Bluestone, J.A. 1995. New perspectives of CD28-B7-mediated T cell costimulation. Immunity. 2:555-559.

16. Thompson, C.B. 1995. Distinct roles for the costimulatory ligands B7-1 and B7-2 in T helper cell differentiation? Cell. 81:979-982.

17. Sayegh, M.H., and Turka, L.A. 1998. The role of T-cell costimulatory activation pathways in transplant rejection. N. Engl. J. Med. 338:1813-1821.
18. Linsley, P.S., and Ledbetter, J.A. 1993. The role of the CD28 receptor during T cell responses to antigen. Annu. Rev. Immunol. 11:191-212.

19. Grewal, I.S., and Flavell, R.A. 1998. CD40 and CD154 in cell-mediated immunity. Annu. Rev. Immunol. 16:111-135.

20. Kaplan, M.H., Sun, Y.L., Hoey, T., and Grusby, M.J. 1996. Impaired IL-12 responses and enhanced development of Th2 cells in Stat4-deficient mice. Nature. 382:174-177.

21. Kaplan, M.H., Schindler, U., Smiley, S.T., and Grusby, M.J. 1996. Stat6 is required for mediating responses to IL-4 and for development of Th2 cells. Immunity. 4:313-319.

22. Corry, R.J., Winn, H.J., and Russell, P.S. 1973. Primarily vascularized allografts of hearts in mice. The role of $\mathrm{H}-2 \mathrm{D}, \mathrm{H}-2 \mathrm{~K}$, and non- $\mathrm{H}-2$ antigens in rejection. Transplantation. 16:343-350.

23. Chen, Z.H. 1991. A technique of cervical heterotopic heart transplantation in mice. Transplantation. 52:1099-1101.

24. Turka, L.A., et al. 1992. T-cell activation by the CD28 ligand B7 is required for cardiac allograft rejection in vivo. Proc. Natl. Acad. Sci. USA. 89:11102-11105.

25. Adams, D.H., Tilney, N.L., Collins, J.J., and Karnovsky, M.J. 1992. Experimental graft arteriosclerosis. I. The Lewis-to-F344 allograft model. Transplantation (Baltimore). 53:1115-1119.

26. Russell, M.E., et al. 1996. Chronic cardiac rejection in the Lewis to F344 rat model: blockade of CD28-B7 costimulation by CTLA4Ig modulates $\mathrm{T}$ cell and macrophage activation and attenuates arteriosclerosis. J. Clin. Invest. 97:833-838.

27. Glysing-Jensen, T., Raisanen-Sokolowski, A., Sayegh, M.H., and Russell, M.H. 1997. Chronic blockade of CD28-B7 mediated T cell costimulation by CTLA4Ig reduces intimal thickening in MHC class I and II incompatible mouse heart allografts. Transplantation. 164:1641-1645.

28. Benichou, G., Valujskikh, A., and Heeger, P.S. 1999. Contributions of direct and indirect $T$ cell alloreactivity during allograft rejection in mice. J. Immunol. 162:352-358.

29. Chen, W., Sayegh, M.H., and Khoury, S.J. 1998. Mechanisms of acquired thymic tolerance in vivo: intrathymic injection of antigen induces apoptosis of thymocytes and peripheral $\mathrm{T}$ cell anergy. J. Immunol. 160:1504-1508.

30. Lin, H., et al. 1998. Cytotoxic T lymphocyte antigen 4 (CTLA4) blockade accelerates the acute rejection of cardiac allografts in CD28-deficient mice: CTLA4 can function independently of CD28. J. Exp. Med. 188:199-204.

31. Hancock, W.W., Buelow, R., Sayegh, M.H., and Turka, L.A. 1998. Antibody-induced transplant arteriosclerosis is prevented by graft expression of anti-oxidant and anti-apoptotic genes. Nat. Med. 4:1392-1396.

32. Iwakoshi, N.N., et al. 2000. Treatment of allograft recipients with donorspecific transfusion and anti-CD154 antibody leads to deletion of alloreactive CD8+ T cells and prolonged graft survival in a CTLA4-dependent manner. J. Immunol. 164:512-521.

33. Piccotti, J.R., et al. 1998. Alloantigen-reactive Th1 development in IL-12deficient mice. J. Immunol. 160:1132-1138.

34. Strom, T.B., et al. 1996. The Th1/Th2 paradigm and the allograft response. Curr. Opin. Immunol. 8:688-693.

35. Piccotti, J.R., Chan, S.Y., VanBuskirk, A.M., Eichwald, E.J., and Bishop, D.K. 1997. Are Th2 helper T lymphocytes beneficial, deleterious, or irrelevant in promoting allograft survival? Transplantation. 63:619-624.

36. Field, E.H., Gao, Q., Chen, N.X., and Rouse, T.M. 1997. Balancing the immune system for tolerance: a case for regulatory CD4 cells. Transplantation. 64:1-7.

37. Khoury, S.J., et al. 1995. CD28-B7 costimulatory blockade by CTLA4Ig prevents actively induced experimental autoimmune encephalomyelitis and inhibits Th1 but spares Th2 cytokines in the central nervous system. J. Immunol. 155:4521-4524.

38. Kuchroo, V.K., et al. 1995. B7-1 and B7-2 costimulatory molecules activate differentially the Th1/Th2 developmental pathways: application to autoimmune disease therapy. Cell. 80:707-718.

39. Oosterwegel, M.A., et al. 1999. The role of CTLA-4 in regulating Th2 differentiation. J. Immunol. 163:2634-2639.

40. Keane-Myers, A., Gause, W.C., Linsley, P.S., Chen, S.J., and Wills-Karp, M. 1997. B7-CD28/CTLA-4 costimulatory pathways are required for the development of $\mathrm{T}$ helper cell 2-mediated allergic airway responses to inhaled antigens. J. Immunol. 158:2042-2049.

41. Larche, M., et al. 1998. Costimulation through CD86 is involved in airway antigen-presenting cell and $\mathrm{T}$ cell responses to allergen in atopic asthmatics. J. Immunol. 161:6375-6382.

42. Padrid, P.A., et al. 1998. CTLA4Ig inhibits airway eosinophilia and hyperresponsiveness by regulating the development of Th1/Th2 subsets in a murine model of asthma. Am. J. Respir. Cell. Mol. Biol. 18:453-462.

43. Harris, N.L., Peach, R.J., and Ronchese, F. 1999. CTLA4-Ig inhibits optimal T helper 2 cell development but not protective immunity or memory response to Nippostrongylus brasiliensis. Eur. J. Immunol. 29:311-316.

44. Corry, D.B., Reiner, S.L., Linsley, P.S., and Locksley, R.M. 1994. Differential effects of blockade of CD28-B7 on the development of Th1 or Th2 effector cells in experimental leishmaniasis. J. Immunol. 153:4142-4148. 
45. Azuma, H., et al. 1996. Blockade of T-cell costimulation prevents development of experimental chronic renal allograft rejection. Proc. Natl. Acad. Sci. USA. 93:12439-12444.

46. Chandraker, A., et al. 1998. Late blockade of T cell costimulation interrupts progression of experimental chronic allograft rejection. J. Clin. Invest. 101:2309-2318.

47. Kaplan, M.H., Wurster, A.L., and Grusby, M.J. 1998. A signal transducer and activator of transcription (Stat)4-independent pathway for the development of T helper type 1 cells. J. Exp. Med. 188:1191-1196.

48. Carter, L.L., and Murphy, K.M. 1999. Lineage-specific requirement for signal transducer and activator of transcription (Stat) 4 in interferon gamma production from CD4(+) versus CD8(+) T cells. J. Exp. Med. 189:1355-1360.

49. Tang, A., Judge, T.A., and Turka, L.A. 1997. Blockade of CD40-CD40 ligand pathway induces tolerance in murine contact hypersensitivity. Eur. J. Immunol. 27:3143-3150.

50. Resetkova, E., et al. 1996. Antibody to gp39, the ligand for CD40 significantly inhibits the humoral response from Graves' thyroid tissues xenografted into severe combined immunodeficient (SCID) mice. Thyroid. 6:267-273.

51. Durie, F.H., et al. 1993. Prevention of collagen-induced arthritis with an antibody to gp39, the ligand for CD40. Science. 261:1328-1330.

52. Li, X.C., Zand, M.S., Li, Y., Zheng, X.X., and Strom, T.B. 1998. On histocompatibility barriers, Th1 to Th2 immune deviation, and the nature of the allograft responses. J. Immunol. 161:2241-2247.
53. Newell, K.A., et al. 1999. Cutting edge: blockade of the CD28/B7 costimulatory pathway inhibits intestinal allograft rejection mediated by CD4+ but not CD8+ T cells. J. Immunol. 163:2358-2362.

54. Trambley, J., et al. 1999. Asialo GM1(+) CD8(+) T cells play a critical role in costimulation blockade-resistant allograft rejection. J. Clin. Invest. 104:1715-1722.

55. Kirk, A.D., et al. 1997. CTLA4-Ig and anti-CD40 ligand prevent renal allograft rejection in primates. Proc. Natl. Acad. Sci. USA. 94:8789-8794.

56. Kirk, A.D., et al. 1999. Treatment with humanized monoclonal antibody against CD154 prevents acute renal allograft rejection in nonhuman primates. Nat. Med. 5:686-693.

57. Larsen, C.P., et al. 1996. Long-term acceptance of skin and cardiac allografts after blocking CD40 and CD28 pathways. Nature. 381:434-438.

58. Wood, K., and Sachs, D.H. 1996. Chimerism and transplantation tolerance: cause and effect [discussion p. 588]. Immunol. Today. 17:584-587.

59. Hamano, K., Rawsthorne, M.A., Bushell, A.R., Morris, P.J., and Wood, K.J. 1996. Evidence that the continued presence of the organ graft and not peripheral donor microchimerism is essential for maintenance of tolerance to alloantigen in vivo in anti-CD4 treated recipients. Transplantation. 62:856-860.

60. Yang, L., DuTemple, B., Gorczynski, R.M., Levy, G., and Zhang, L. 1999. Evidence for epitope spreading and active suppression in skin graft tolerance after donor-specific transfusion. Transplantation. 67:1404-1410. 